\title{
Proposta de Aplicação e Avaliação de Conceitos do Pensamento Computacional em Crianças Hospitalizadas
}

\author{
Jussara S. Oliveira Zimmermann ${ }^{1}$, Andreia Watanabe², Thiago Schumacher \\ Barcelos $^{3}$, Felipe Mancini ${ }^{1}$ \\ ${ }^{1}$ Gestão e Informática em Saúde - Universidade Federal de São Paulo, São Paulo \\ (UNIFESP) São Paulo - SP - Brasil \\ ${ }^{2}$ Instituto da Criança do Hospital das Clínicas da Faculdade de Medicina da \\ Universidade de São Paulo (HCFMUSP) - São Paulo - SP - Brasil \\ ${ }^{3}$ Instituto Federal de Educação, Ciência e Tecnologia de São Paulo (IFSP) - Guarulhos

$$
-\mathrm{SP} \text { - Brasil }
$$ \\ \{jussara.zimmermann@unifesp.br, a.watanabe@hc.fm.usp.br, \\ tsbarcelos@ifsp.edu.br, fmancinieunifesp.br
}

\begin{abstract}
Resumo. O estudo objetiva avaliar o impacto da utilização do software ScratchJr para a aprendizagem de conceitos do Pensamento Computacional e sua influência sobre o desempenho cognitivo em crianças hospitalizadas. Desenvolveremos oficinas, onde sujeitos da pesquisa utilizarão a arquitetura computacional para a criação de jogos e animações. Para verificar o aprimoramento sobre conceitos do Pensamento Computacional serão utilizados análise de artefatos e entrevista ao final das oficinas. Para avaliar o nível intelectual dos participantes utilizaremos a escala WISC-III pré e pós realização das oficinas.
\end{abstract}

\begin{abstract}
The study aims to evaluate the impact of using ScratchJr software for learning Computational Thinking concepts and its influence on cognitive performance in hospitalized children. We aim to develop workshops where research subjects will use this computational architecture for creating games and animations. In order to verify the improvement of Computational Thinking concepts, an analysis of artifacts and an interview at the end of the workshops will be performed. To assess the intellectual level of the participants we will use the WISC-III scale before and after the offering of the workshops.
\end{abstract}

\section{Introdução}

A identidade infantil, muitas vezes, é diluída numa situação de internação em que a criança está inserida numa realidade diferente da sua rotina. Sua autonomia pode ser sufocada pelas práticas hospitalares que tratam a criança como paciente, como aquele que inspira e necessita de cuidados médicos e que parece alheio aos acontecimentos ao seu redor. O trabalho pedagógico em hospitais apresenta diversas interfaces de atuação e possui diferentes olhares que investigam a compreensão e a construção de um modelo de atuação. No entanto, é preciso deixar claro que tanto a educação não é elemento exclusivo da escola quanto a saúde não é elemento exclusivo do hospital (Fontes, 2005).

O hospital também é considerado um centro de educação (Fontes, 2005) e segundo definição do Ministério da Saúde, "é parte integrante de uma organização médica e social, cuja função básica consiste em proporcionar à população assistência médica integral, curativa e preventiva, sob quaisquer regimes de atendimento, inclusive o domiciliar, 
constituindo-se também em centro de educação, capacitação de recursos humanos e de pesquisas em saúde, bem como de encaminhamento de pacientes, cabendo-lhe supervisionar e orientar os estabelecimentos de saúde a ele vinculados tecnicamente" (Brasil, 1997).

Atualmente, o crescente uso das redes sem fio e de dispositivos móveis permite aos seus usuários o acesso a serviços e dados independentemente de sua localização física. A possibilidade de levar consigo o objeto de estudo, ou poder acessá-lo de qualquer lugar, potencializa o uso de dispositivos móveis na educação. Esta abordagem é definida como Aprendizagem com Mobilidade (Roschelle, Jeremy e Pea, 2002).

Para Piaget, a aprendizagem é, em geral, provocada por situações externas ao sujeito e só acontece quando há assimilação ativa por parte deste. A ênfase é colocada na atividade do próprio sujeito, ou seja, a importância da sua ação frente à atividade é o que o responsabiliza pelo seu próprio processo de aprender (Piaget, 1972).

Em 2006, Wing sistematizou uma abordagem para resolução de problemas que combinou o pensamento crítico com os fundamentos da computação, definida como Pensamento Computacional. Esse método utiliza os fundamentos e técnicas da Ciência da Computação para a solução de problemas. A base dessa metodologia consiste na utilização do computador como um instrumento para melhor compreensão do mundo atual, permeado por dispositivos computacionais, refletindo positivamente em na produtividade, inventividade e criatividade do usuário (Wing, 2006).

O conceito de Pensamento Computacional se refere ao domínio de competências e habilidades da Computação que podem ser aplicadas à compreensão de conteúdos de outras áreas da ciência. A tecnologia computacional, que permeia nosso mundo e rotinas, é vista como importante recurso provedor de subsídios para que os indivíduos possam não apenas utilizar a tecnologia, mas também compreendê-la e serem capazes de implementar soluções para problemas utilizando recursos computacionais. Especificamente, o pensamento computacional pode auxiliar na resolução de problemas das mais diversas áreas, através de conceitos como abstração, decomposição, entre outros. Além disso, pode liderar a busca pelo aperfeiçoamento das tecnologias ligadas à informação e comunicação (CSTA, 2011a).

Como o Pensamento Computacional amplia a capacidade de dedução e resolução de problemas, muitos estudiosos acreditam que seus conceitos e metodologia deveriam ser ensinados desde cedo em escolas primárias (Sica, 2008). Além da competência para a leitura, escrita e aritmética, o Pensamento Computacional deveria ser adicionado ao currículo escolar para que a função de ampliação da capacidade analítica-computacional durante a formação das crianças seja alcançada de forma mais efetiva. A Microsoft e a Universidade de Carnegie Mellon criaram o Centro de Pensamento Computacional em 2007 e a Google tem se empenhado em promover esta metodologia em todo o currículo do ensino primário e secundário nos Estados Unidos (Carvalho e Moro, 2013).

No Brasil, atualmente, esses conceitos são aprendidos somente por aqueles que optam por cursos técnicos ou de graduação na área da computação. Esse fato se contrapõe às exigências atuais do mercado de trabalho, onde grande parte das profissões exige uma compreensão da Ciência da Computação. Esse é o caso das áreas ligadas à arte e entretenimento, comunicação, saúde, entre outros. Assim, o Pensamento Computacional mostra-se como uma das habilidades mais importantes e menos compreendidas que se tornam necessárias para o pleno exercício da cidadania neste século (Blikstein, 2008). 
Com o Pensamento Computacional os usuários passam a ter não só a capacidade de desenvolver os seus próprios sistemas, como o reforço de competências adjacentes, sendo elas: o pensamento abstrato (utilização de diferentes níveis de abstração para perceber os problemas e, passo a passo, soluciona-los), o pensamento algorítmico (expressão de soluções em diferentes passos de forma a encontrar a forma mais eficaz e eficiente de resolver um problema), o pensamento lógico (formulação e exclusão de hipóteses) e o pensamento dimensionável (decomposição de um grande problema em pequenas partes ou composição de pequenas partes para formular uma solução mais complexa) (CSTA, 2011b).

Dessa forma, a introdução de conceitos de Ciência da Computação na educação é apontada como um importante recurso para o aprimoramento do raciocínio computacional dos indivíduos, pelo seu caráter transversal a todas as ciências (Nunes, 2011). De acordo com Douglas Rushkoff, "aprender uma linguagem de programação nos dias atuais é tão importante quanto aprender a ler e escrever, pois, na chamada sociedade da informação, a distância entre usar um programa e criar um tornou-se cada vez mais ampla, a ponto de as pessoas não saberem mais o que está acontecendo atrás da tela do computador; portanto qualquer pessoa que saiba criar um programa de computador será capaz de criar a realidade em que o restante estará inserido" (Rushkoff, 2012).

Assim, pretendemos utilizar um ambiente de programação disponível para dispositivos móveis para avaliar o impacto de uma sequência didática baseada na construção de jogos digitais que contribua com o desenvolvimento de elementos do Pensamento Computacional e com o desempenho cognitivo em crianças hospitalizadas. $\mathrm{Na}$ sequência do artigo são apresentadas a metodologia do estudo, na Seção 2, e os resultados esperados na Seção 3.

\section{Metodologia}

O projeto relatado no presente documento será realizado com pacientes da Unidade de Nefrologia Pediátrica de um hospital de alta complexidade, que são submetidos à procedimentos para filtragem do sangue, que possui duração de 3 horas, no mínimo 3 vezes por semana. O estudo envolverá 10 pacientes entre 6 e 12 anos. A formação do grupo de participantes se dará por adesão do público-alvo a este estudo.

Utilizaremos o tipo de pesquisa descritiva, no formato de estudo de caso, com abordagem qualitativa. Inicialmente os pacientes participantes do projeto responderão a um questionário de avaliação de perfil dos usuários para verificar dados sobre seu perfil sócio demográfico, informações referentes à utilização de dispositivos computacionais e interesses relacionados a jogos digitais. Essas informações serão de fundamental importância para o desenvolvimento das etapas da oficina que terão como base de enredo as preferências dos participantes do estudo.

Na sequência, aplicaremos individualmente e em local reservado, a Escala de Inteligência Wechsler para Crianças (WISC-III) (Wechsler, 1964), que visa avaliar o nível intelectual de indivíduos de 6 a 15 anos e 11 meses. A escala é formada por diversos subtestes que avaliam diferentes aspectos da inteligência, desenvolvidos levando em consideração a concepção da inteligência como a capacidade do indivíduo em raciocinar, lidar e operar com propósito, racionalmente e efetivamente com o seu meio ambiente. Aplicaremos todos os subtestes dessa escala que possuem como objetivo investigar capacidades mentais diferentes, mas que juntas, oferecem uma estimativa da capacidade intelectual geral da criança (Cruz, 2005). 
O cronograma de atividades das oficinas foi dividido em 10 encontros individuais, onde os pacientes realizarão desafios relacionados com a aprendizagem de conceitos do Pensamento Computacional. Para tal utilizaremos o software ScratchJr como ferramenta computacional para o desenvolvimento das tarefas propostas. Por meio da utilização desse ambiente de programação é possível desenvolver histórias interativas, animações, jogos, músicas e artes. O ScratchJr constitui-se de uma linguagem de programação e ambiente de desenvolvimento de software gratuito, desenvolvido pelo Instituto de Tecnologia de Massachusetts (Manzano e Oliveira, 2008). A equipe do ScratchJr desenhou o idioma da interface e programação para combinar, o desenvolvimento pessoal, social, emocional e cognitivo de crianças acima de 5 (cinco) anos (Flannery, 2009).

Em cada atividade da oficina, realizada 2 vezes por semana, os pacientes receberão instruções sobre os objetivos propostos para as tarefas dos encontros. Serão apresentados a um exemplo da tarefa proposta sendo executada e a partir daí iniciam o trabalho. O pesquisador atuará como um facilitador, observando o trabalho e intervendo quando os pacientes solicitarem esclarecimentos. Na Tabela 1 apresentamos um resumo dos conceitos do Pensamento Computacional a serem abordados durante os encontros das Oficinas.

Tabela 1. Atividades das Oficinas

\begin{tabular}{|l|l|}
\hline Atividade & Conceitos do Pensamento Computacional a serem abordados \\
\hline Oficina 1 & Familiarização do ambiente e sequência \\
\hline Oficina 2 & Construção de jogos usando sequência \\
\hline Oficina 3 & Conceito de operadores e atividades usando sequência e operadores \\
\hline Oficina 4 & Construção de jogos usando sequência e operadores \\
\hline Oficina 5 & Conceito de condicional e atividades usando sequência, operadores e condicional \\
\hline Oficina 6 & Construção de jogos usando sequência, operadores e condicional \\
\hline Oficina 7 & Conceito de laços e atividades usando sequência, operadores, condicional e laços \\
\hline Oficina 8 & Construção de jogo com a utilização todos os conceitos aprendidos \\
\hline Oficina 9 & Construção de jogo com a utilização todos os conceitos aprendidos \\
\hline Oficina 10 & Construção de jogo com a utilização todos os conceitos aprendidos \\
\hline
\end{tabular}

O jogo estará disponível para que a criança o utilize em um tablete durante o período de permanência no hospital para a realização da hemodiálise.

Ao final dos 10 encontros aplicaremos a avaliação do experimento utilizando novamente a escala WISC-III, além de uma Avaliação Baseada em Evidências e uma Entrevista Baseada em Evidências e Artefatos. A Avaliação Baseada em Evidências preconiza a observação, registro e análise da utilização das ferramentas que apontam o uso de conceitos do Pensamento Computacional durante a elaboração dos projetos dos usuários no ScratchJr. No entanto, essa ferramenta de avaliação é totalmente orientada para produto e por esse motivo não revela a intencionalidade por trás do processo de desenvolvimento de projeto, e consequentemente oculta informações sobre conceitos do pensamento computacional que podem ter sido utilizados (Brennan e Resnick, 2012).

Dessa forma, a Entrevista Baseada em Evidências e Artefatos complementa esse instrumento avaliativo ao passo que explora fatores motivadores e a intencionalidade dos usuários possibilitando o levantamento de questões que apoiam o conhecimento sobre o processo de desenvolvimento do projeto, tais como: como começou, como o projeto evoluiu durante o desenvolvimento, o que era importante para eles saber para desenvolver os artefatos propostos, qual a intenção final do projeto, os problemas que ele encontrou 
V Congresso Brasileiro de Informática na Educação (CBIE 2016)

Anais dos Workshops do V Congresso Brasileiro de Informática na Educação (CBIE 2016)

durante todo o processo e como ele lidou com esses problemas. Assim, a Entrevista Baseadas em Evidências e Artefatos permite o melhor entendimento sobre a fluência do usuário sobre conceitos particulares, a expansão do foco do produto exclusivamente para incluir processo permitindo a identificação da utilização de práticas pensamento computacional durante o desenvolvimento de projetos (Brennan e Resnick, 2012).

No presente estudo realizaremos as entrevistas imediatamente após a realização da tarefa proposta, o que contribuirá para a riqueza de detalhes quanto a intencionalidade durante a criação dos projetos.

\section{Contribuições esperadas}

Neste estudo apontamos como contribuições científicas e tecnológicas o levantamento de informações sobre perfil sociodemográfico, utilização de dispositivos computacionais, os interesses e fluências relacionados à jogos digitais dos sujeitos de pesquisa e a avaliação do impacto da utilização de um ambiente de programação para dispositivos móveis na aprendizagem de conceitos do Pensamento Computacional e o impacto dessa aprendizagem sobre o desempenho cognitivo de crianças hospitalizadas participantes do estudo.

Ressaltamos que toda criança e adolescente tem direito a viver num ambiente favorecedor de seu processo de desenvolvimento, que lhe ofereça segurança, apoio, proteção e cuidado. Os interesses dessa nova geração estão cada vez mais ligados ao mundo virtual que, nos últimos vinte e cinco anos, vem crescendo e avançando a passos largos no desenvolvimento de mundos virtuais, estórias interativas, comunidades de jogadores de jogos on-line, e temas abrangendo uma vasta quantidade de experiências humanas. Dessa forma, também destacamos as seguintes contribuições sociais para essa pesquisa:

1 - Desenvolvimento da aprendizagem de diferentes conteúdos e informações que facilitarão no processo de aprendizagem e tomada de decisão para as crianças participantes da proposta.

2 - Aprimoramento das práticas e ferramentas utilizadas para o estímulo, motivação e desenvolvimento da aprendizagem em crianças hospitalizadas, alinhadas às exigências sociais do contato constante com ferramentas computacionais e avanços tecnológicos.

3 - Motivação das crianças que, ao brincarem, estarão desenvolvendo aprendizagens relativas aos conceitos do Pensamento Computacional e da resolução de problemas que contribuirão para o desenvolvimento e formação de conhecimentos importantes que serão levados ao longo da vida dos pacientes.

\section{Referências}

Brasil. Ministério da Saúde. Definições e Normas das instituições e serviços de saúde. Diário Oficial da União de 5/4/1977 - Seção I, Parte I, p. 3929.

Blikstein, Paulo. O pensamento computacional e a reinvenção do computador na educação, 2008. Disponível em <http://www.blikstein.com/paulo/documents/ online/ol_pensamento_computacional.html>. Acesso em: 05 set. 2011 
V Congresso Brasileiro de Informática na Educação (CBIE 2016)

Anais dos Workshops do V Congresso Brasileiro de Informática na Educação (CBIE 2016)

Brennan, K.; Resnick, M. New frameworks for studying and assessing the development of computational thinking. In: AERA 2012, 2012, Vancouver. Proceedings of the 2012 annual meeting of the American Educational Research Association. Vancouver: American Educational Research Association, 2012.

de Carvalho, Márcio Luiz Bunte; Chaimowicz, Luiz; Moro, Mirella M. Pensamento Computacional no Ensino Médio Mineiro. In: Workshop de Educação em Informática (WEI), 2013, Maceió. Anais do XXXIII Congresso da Sociedade Brasileira de Computação, 2013.

Cruz, Maria Beatriz Zanarella. WISC III: escala de inteligência Wechsler para crianças: manual. (2005): 199-201.

Flannery, Louise P., et al. Designing ScratchJr: Support for early childhood learning through computer programming. Proceedings of the 12th International Conference on Interaction Design and Children. ACM, 2013.

Fontes, Rejane de S. A escuta pedagógica à criança hospitalizada: discutindo o papel da educação no hospital. Revista Brasileira de Educação, 29.2, p. 119-139, 2005.

Manzano, José Augusto N. G; Oliveira, Jayr Figueiredo de. Algoritmos: lógica para desenvolvimento de programação de computadores. 22. ed. São Paulo: Érica, 2009. $240 \mathrm{p}$.

Marques, T. M. O Scratch dá o que falar: dos usos domésticos aos usos na escola. Revista Educação e Matemática, Lisboa, número 96, 44-48, 2008.

Nunes, Daltro José. Ciência da computação na educação básica. Jornal da Ciência, v. 9, 2011.

Piaget, Jean. Development and learning. In: LAVATELLY, C.; STENDLER, F. Reading in child behavior and development. Nova York: Hartcourt Brace Janovich, 1972.

Rushkoff, D. As 10 questões essenciais da era digital. São Paulo: Editora Saraiva, 2012. Disponível em: <http://www.hipertextus.net/volume8/09-Hipertextus-Vol8-AcirMario-Karwoski.pdf> . Acesso em: 30 nov. 2015.

Sica, Carlos. Ciência da Computação no Ensino Básico e Médio, 2008. Disponível em $<$ http://blogs.odiario.com/carlossica/2011/10/07/ciencia-da-computacao-no-ensinomedio>. Acesso em: 22 ago. 2015.

The CSTA Standards Task Force. CSTA K-12 Computer Science Standards. ACM Computer Science Teachers Association, New York, 2011.

The CSTA Standards Task Force. A Model Curriculum for K-12 Computer Science: Report of the ACM K-12 Task Force Computer Science Curriculum Committee, 2011. Disponível em <http://www.csta.acm.org/Curriculum/sub/CurrFiles/CSTA_K12_CSS.pdf $>$. Acesso em: 01 ago. 2015.

Wechsler, D. (1964). Escala de Inteligência para Crianças - WISC - Manual de aplicação e cotação (A. M. Poppovic, Trad.). Rio de Janeiro: CEPA.

Wing, Jeannette M. Computational thinking. Communications of the ACM, v. 49, n.3, 2006: 33-35 